\title{
Development of photonic and thermodynamic crystals conforming to sustainability conscious materials tectonics
}

\author{
S. Kirihara, N. Ohta, T. Niki, Y. Uehara \& S. Tasaki \\ Joining and Welding Research Institute, Osaka University, Japan
}

\begin{abstract}
Photonic crystals with periodic variations in dielectric constants can exhibit band gaps in transmission spectra of electromagnetic waves through Bragg diffractions. Introduced structural defects can confine the electromagnetic energies at specific wavelengths, and localized modes of transmission peaks can be formed in above mentioned forbidden gaps. Moreover, thermodynamic crystals with periodically percolated morphologies of metals and ceramics can realize uniform dispersions in thermal and kinetic energies effectively. Introduced artificial dislocations or graded structures into the ideal periodicities realize heat and stress flow controls intentionally. These artificial crystals were processed by micro stereolithography. The electromagnetic wave transmittances, thermal conductivities and mechanical properties for the formed photonic and thermodynamic crystals were measured and compared with simulated results. The photonic and thermodynamic crystals will be applied to novel electromagnetic wave resonators and mechanical tools, respectively. Effective energy flow control with reducing losses will be realized along sustainability conscious strategies of artificial materials tectonics.
\end{abstract}

Keywords: photonic crystal, thermodynamic crystal, stereolithography.

\section{Introduction}

Photonic crystals with periodic arrangements of dielectric lattices can form forbidden gaps in electromagnetic waves spectra $[1,2]$. These artificial crystals can totally reflect the light or electromagnetic waves at wavelengths comparable to the lattice spacing by Bragg deflection. The introduced structural defects in the periodic arrangements can localize the electromagnetic wave energies and 
form the transmission mode peaks in the band gaps according to the sizes and dielectric constants of the defects. Recently, we have newly developed micro stereolithography system to fabricate the micro photonic crystals composed of the ceramic lattices for the electromagnetic wave control in terahertz frequency ranges $[3,4]$. In the near future, the terahertz wave will be expected to apply to various types of novel sensors which can detect gun powders, drugs, bacteria in foods, micro cracks in electric devices, cancer cells in human skin and other physical, chemical and living events [5, 6]. In this study, the ceramic lattices with diamond structures were created to realize the perfect band gap formations opening for all crystal directions [7, 8]. Subsequently, we successfully fabricated twinned diamond structures with plane defects between the mirror symmetric lattice patterns [9-11]. Localized modes to transmit the terahertz wave selectively were formed in the band gap region. Transmission spectra in the terahertz range through the alumina lattices were measured. The selective transmission modes in the band gaps were observed for the twinned diamond structures, and localized mode behaviours of the terahertz waves in the plane defects will be discussed.

Thermodynamic crystals with periodic arrangements of ceramic and metal phases were fabricated artificially to control heat and stress flows intentionally by computer aided design, manufacturing and evaluation [12]. Graded structures in volume fractions of ceramic and metal phases were created successfully to realize directional controls of heat and stress energies distributions. In this study, the alumina structures including air spheres with body centered cubic patterns were processed by using the micro stereolithography, and resin slurries with pure copper particles were infiltrated into the ceramics objects. Through dewaxing and sintering treatments, the thermodynamic crystals with the metals spheres arrangements in the ceramics matrices could be obtained exactly. Moreover, the functionally graded materials with continuous variations in volume fractions of the ceramics and metals were fabricated. The intermediate regions with the percolated morphologies were formed between the copper and alumina layers. The artificial microstructures composed of the alumina and pure copper phases were observed by using a digital optical microscope and a scanning electron microscope. The part accuracies of the periodic structures are measured. Thermal and mechanical properties were theoretically simulated method. The directional propagations of the heat and stress energy flows in the periodically percolated materials morphologies will be considered theoretically.

\section{Experimental procedure}

The photonic and thermodynamic crystals with the diamond lattice and body center cubic structure were spatially modelled by a computer graphic application (Think Design: Toyota Keram, Japan) as shown in Figures 1 and 2, respectively. In the case of diamond photonic crystal, the lattice constant was designed as $1 \mathrm{~mm}$. The whole structure was $6 \times 6 \times 2 \mathrm{~mm}^{3}$ in size, consisting of 72 unit cells. The aspect ratio of the lattices was adjusted to be 1.5 . In the case of thermodynamic crystal, the inverse model of $10 \times 10 \times 10 \mathrm{~mm}$ in size included the 


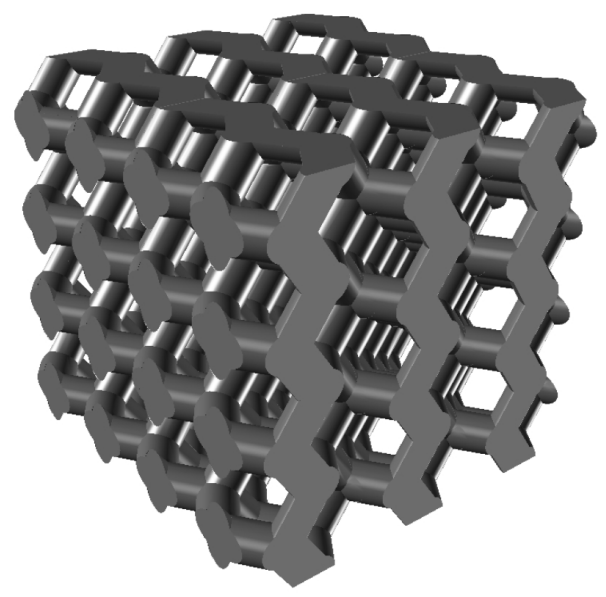

Figure 1: A computer graphic model of photonic crystal with diamond lattice arrangements to create periodic variations of dielectric constants.

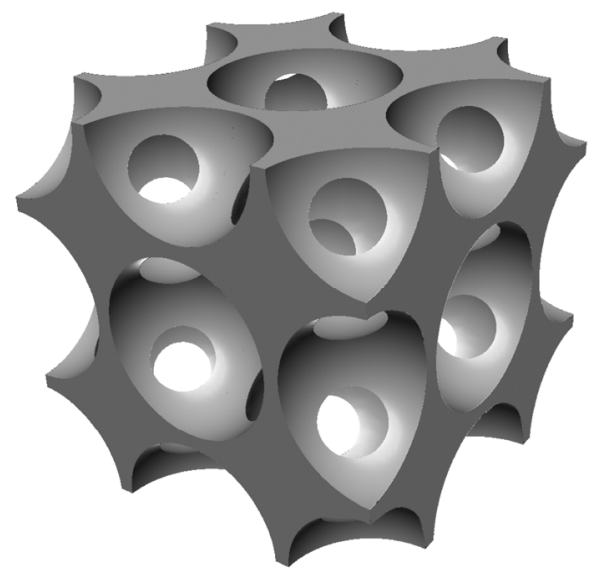

Figure 2: The computer graphic model of thermodynamic crystal with inverse sphere arrangements to create a body centered cubic structure.

air spheres of $4.0 \mathrm{~mm}$ in diameter. The lattice spacing for $<100>$ direction was $4.5 \mathrm{~mm}$. The volume ratio of the matrix was $26.5 \%$. These designed models were converted into the numerical data of stereolithographic format and sliced into thin sections.

In the fabrication process of the photonic crystals, a micro pattering stereolithography system (SI-C1000: D-MEC, Japan) was used as shown in Figure 3. Photo sensitive acrylic resins dispersed with alumina particles of $170 \mathrm{~nm}$ in diameter at 40 volume \% were fed over substrates from dispenser nozzles. The highly viscous resin paste was fed with controlled air pressure. It was spread uniformly by moving a knife edge. The thickness of each layer was controlled to 
$10 \mu \mathrm{m}$. A two dimensional pattern was formed by illuminating visible laser of $405 \mathrm{~nm}$ in wavelength on the resin paste surface. The high resolution has been achieved by using a digital micro mirror device (DMD) and an objective lens. Figure 3 shows a schematic of the micro stereolithography system. The DMD is an optical element assembled by micro mirrors of $14 \mu \mathrm{m}$ in edge length. The tilting of each tiny mirror can be controlled according to the two dimensional cross sectional data transferred for a computer. The three dimensional structures were built through stacking these patterns. In order to avoid deformation and cracking during dewaxing, careful investigation for the heat treatment processes were required. The precursors with diamond structures were heated at various temperatures from 100 to $600^{\circ} \mathrm{C}$ while the heating rate was $1.0^{\circ} \mathrm{C} / \mathrm{min}$. The dewaxing process was observed in respect to the weight and color changes. The alumina particles could be sintered at 1500 or $1000^{\circ} \mathrm{C}$, respectively. The heating rate was $8.0^{\circ} \mathrm{C} / \mathrm{min}$. The density of the sintered sample was measured by the Archimedes method. The microstructures were observed by optical microscope and scanning electron microscopy (SEM). The transmittance and the phase shift of incident terahertz waves were measured by using terahertz time domain spectroscopy (TDS) device (J-Spec2001: Aispec, Japan). The measured terahertz properties were compared with theoretical simulations by using a transmission line modeling (TLM) program (Micro-Stripes: Flomerics, EU).

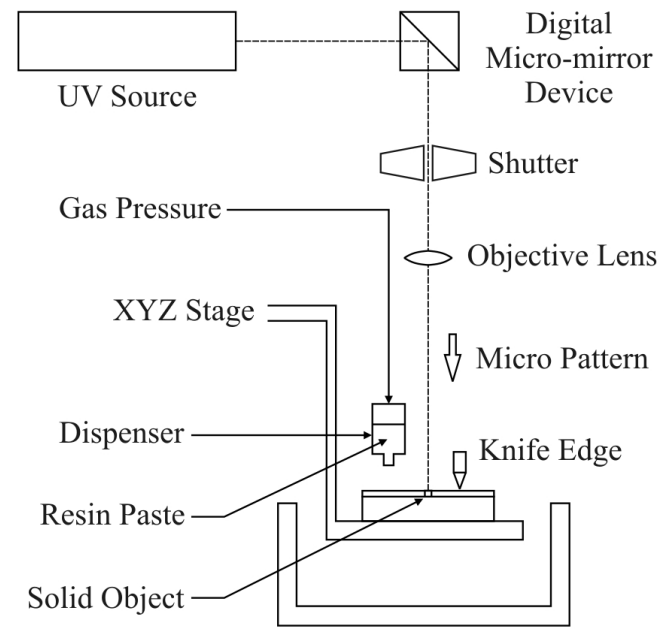

Figure 3: A schematic illustration of a micro pattering stereolithography by using continuous exposure techniques of ultra violet fine images.

The ceramics models of the inversed body centered cubic structures were fabricated automatically by utilizing a laser scanning stereolithographic machine (SCS-300P: D-MEC, Japan) as shown in Figure 4. The alumina particles of $1.8 \mu \mathrm{m}$ in average diameter were dispersed into the photo sensitive acrylic resin at $70 \%$ in volume contents. The composite resin paste was spread on a grass 
substrate with $50 \mu \mathrm{m}$ in layer thickness by the mechanical knife edge. An ultraviolet laser beam of $355 \mathrm{~nm}$ in wavelength and $100 \mu \mathrm{m}$ in spot diameter was scanned on the resin surface to create two dimensional images with $10 \mu \mathrm{m}$ in part accuracy. Through the layer stacking, sub-millimeter order three dimensional composite models were formed successfully. These obtained precursors were dewaxed and sintered at 600 and $1500^{\circ} \mathrm{C}$ for 2 hs with the heating rate of 1 and $8^{\circ} \mathrm{C} / \mathrm{min}$, respectively. Subsequently, the polyester resin past mixed with the pure copper particles of $75 \mu \mathrm{m}$ in average diameter at $55 \%$ in volume contents were percolated into the inverse ceramic structures. After the dewaxing for the surly infiltrated samples at $800^{\circ} \mathrm{C}$ for $2 \mathrm{hs}$ with heating rate of $1{ }^{\circ} \mathrm{C} / \mathrm{min}$, the pure copper particles were sintered at $1000^{\circ} \mathrm{C}$ for $2 \mathrm{hs}$ with heating rate of $8^{\circ} \mathrm{C} / \mathrm{min}$ in an argon atmosphere. The densities of the sintered samples were measured by Archimedes method. The part accuracy of the metal spheres arrangements in the ceramic matrices were measured by using the digital optical microscope (DOM) system (VHX-200: Keyence, Japan). The microstructures of ceramic and metal phases were closely observed by using the SEM. The heat and stress flows were simulated theoretically in the thermodynamic crystals by using a finite element method (FDTD) application (Ansys: Cybernet Systems, Japan).

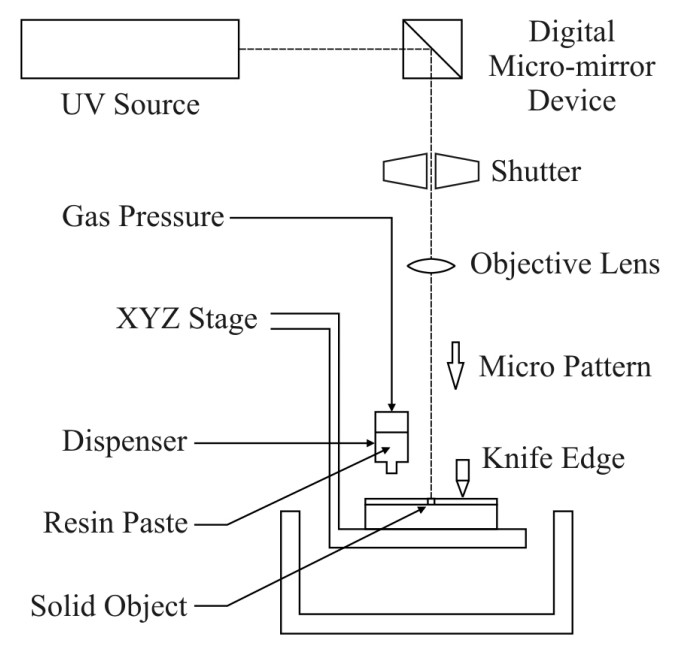

Figure 4: The schematic illustration of a stereolithography system by using ultra violet laser scanning techniques of cross sectional patterns.

\section{Results and discussion}

The lattice structures composed of the alumina dispersed acrylic resin were processed exactly by using the micro stereolithography. The spatial resolution was approximately $0.5 \%$. Figure 5 shows a (100) plane of the sintered diamond structure composed of the micrometer order alumina lattice. The deformation and cracking were not observed. The linear shrinkage on the horizontal axis was 


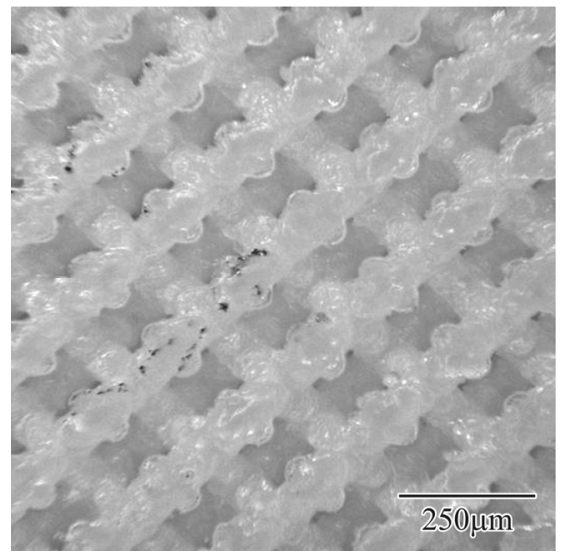

Figure 5: A micro photonic crystal composed of alumina ceramic lattices with the diamond structure fabricated by using the stereolithography.

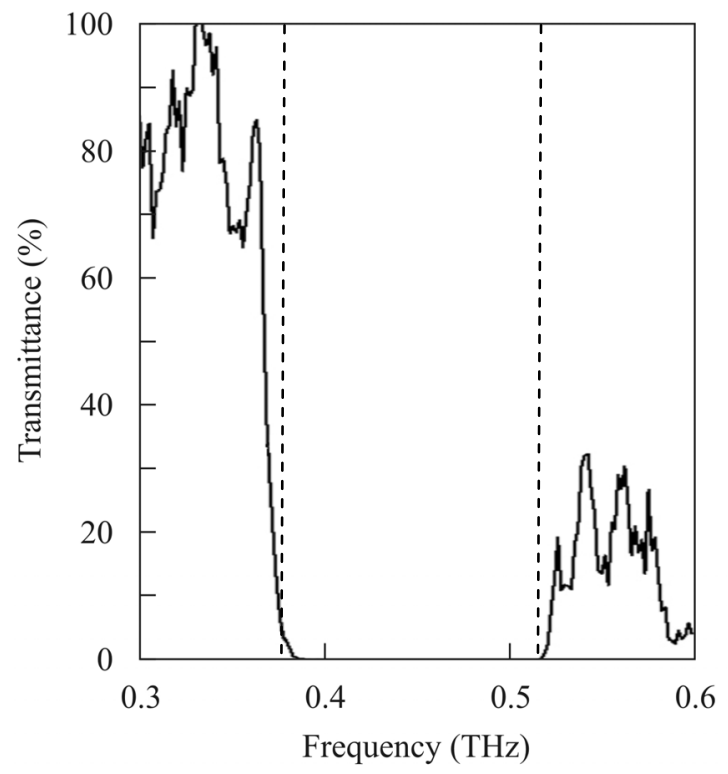

Figure 6: A measured transmission spectrum of terahertz waves for the $\Gamma-\mathrm{X}$ $<100>$ direction in the micro alumina photonic crystal.

$23.8 \%$ and that on the vertical axis was $24.6 \%$. The relative density reached $97.5 \%$. Dense alumina microstructure was formed, and the average grain size was approximately $2 \mu \mathrm{m}$. The measured dielectric constant of the lattice was about 10. The terahertz wave attenuation of the transmission amplitude through the alumina diamond structure for the $\Gamma-\mathrm{X}<100>$ crystal direction is shown in 
Figure 6. The forbidden gap is formed at the frequency range of from 0.37 to $0.52 \mathrm{THz}$. The dotted lines show the higher and lower band gap edges calculated by using the TLM method. The similar transmission spectra for the $\Gamma-K<110>$ and $\Gamma$-L $<111>$ directions were obtained. These measured results of the photonic band gap frequencies were verified to have good agreements with the simulated

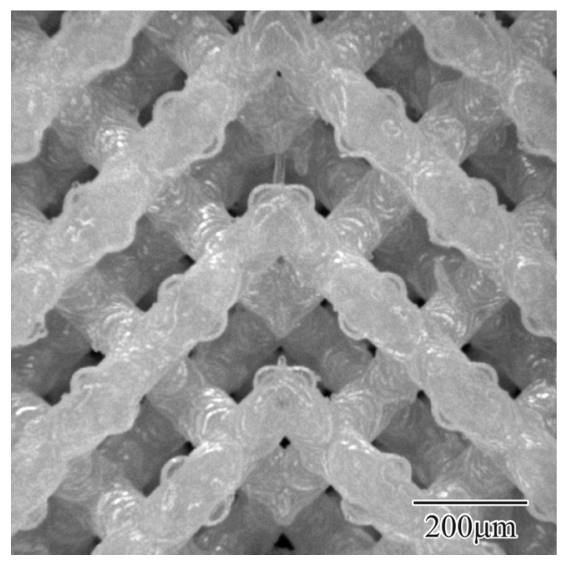

Figure 7: The twinned diamond photonic crystal with a plane defect between mirror symmetric lattices parallel to the (100) layer stacking.

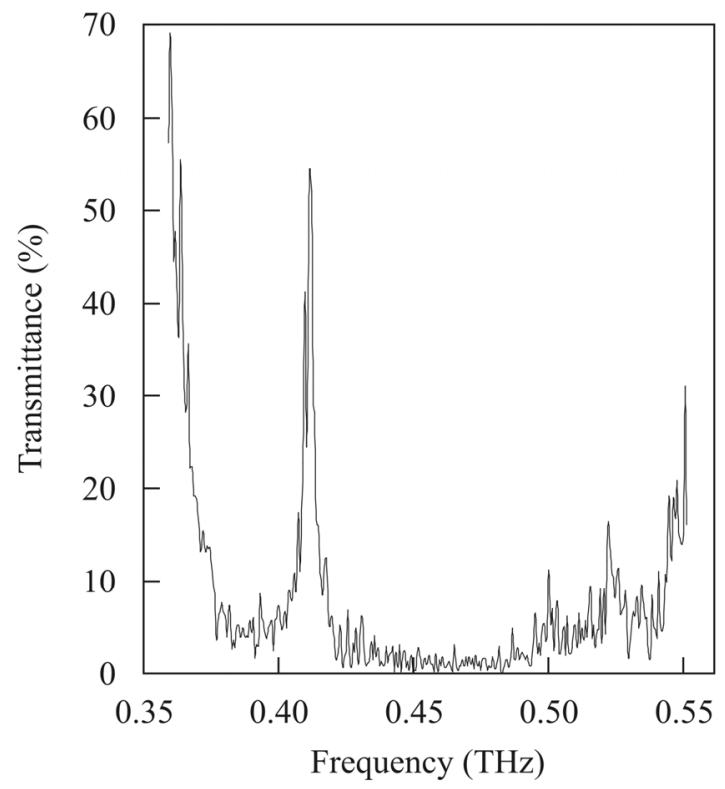

Figure 8: A localized mode formed in the photonic band gap range through the twinned diamond structure of the alumina lattices. 
ones within a tolerance of 5\%. A common region of the band gaps was observed in every crystal direction at the frequency range from 0.40 to $0.47 \mathrm{THz}$, where the electromagnetic wave cannot transmit through the lattices. Figure 7 shows the twinned diamond structure composed of mirror symmetric alumina lattices. The plane defect forms parallel to the (100) crystal layers. The transmission spectrum for the $\Gamma-\mathrm{X}<100>$ crystal direction of the twinned diamond structure is shown in Figure 8. The localized mode forms in the photonic band gap. At the peak frequency, the incident terahertz wave localized strongly in the plane defect, and the amplified wave propagated to the opposite side. The photonic
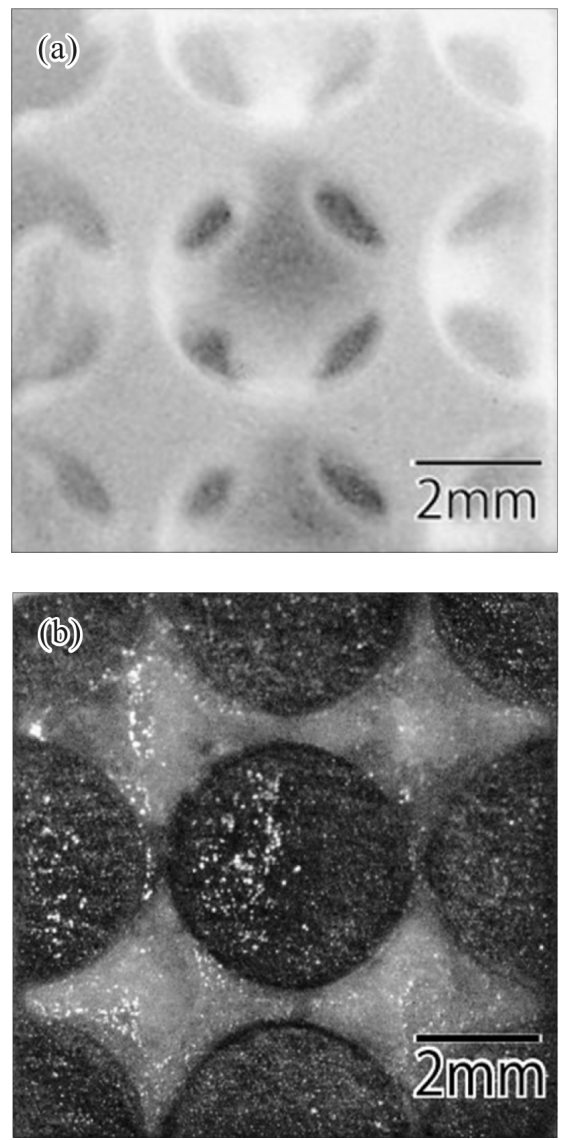

Figure 9: A sintered alumina lattice with the inverse body centered cubic structure fabricated by the stereolithography of a structural joining process (a) and a fabricated thermodynamic crystal with periodic arrangements of pure copper spheres in an alumina matrix through powder sintering treatments (b). 
crystal with the twinned diamond structure to form the band gap and the localized mode will be applied to the effective terahertz wave antennas, resonators and filters.

The inverse models composed of the alumina dispersed acrylic resin were processed successfully by using the stereolithography. The spatial resolution was approximately $0.5 \%$ in size. Figure 9 (a) shows (100) planes of the sintered body centered cubic structure composed of the sub-millimeter order alumina bulk including the air spheres. The deformation and cracking were not observed. The linear shrinkage on the horizontal axis was $6.5 \%$ and that on the vertical axis was $7.8 \%$. It is possible to obtain the uniform shrinkage by designing an appropriate elongated structure in the vertical direction for compensation to the gravity effect. The measured relative density reached 98.5\%. Dense alumina microstructure was formed, and the average grain size was approximately $2 \mu \mathrm{m}$. Figure 9(b) shows the (100) plane in the sintered thermodynamic crystal model composed of the pure copper spheres and alumina matrix. Diffraction peaks indicating the formation of copper oxide phases did not appeared in X-ray diffraction spectra. Cracks and pores as the sintering defects were not observed in the copper phase microstructures. The smooth interfaces between the copper spheres and alumina matrix were obtained successfully. These material phases were joined continuously without intermediate layers. Three dimensional heat and stress distributions in the fabricated thermodynamic crystals were simulated as shown in Figure 10(a) and (b), respectively. The heat flow transmitted from the source plane to the opposite side through the connected copper spheres of the percolated metal phases with high thermal conductivities. Compare with this, the tensile stress were distributed periodically into the alumina lattice parts of the distributed ceramics phases with high Young's modulus. Through introductions of point or plane defects in to the perfect periodic structures, heat and stress distributions can be localized into the specific regions to control the thermal and

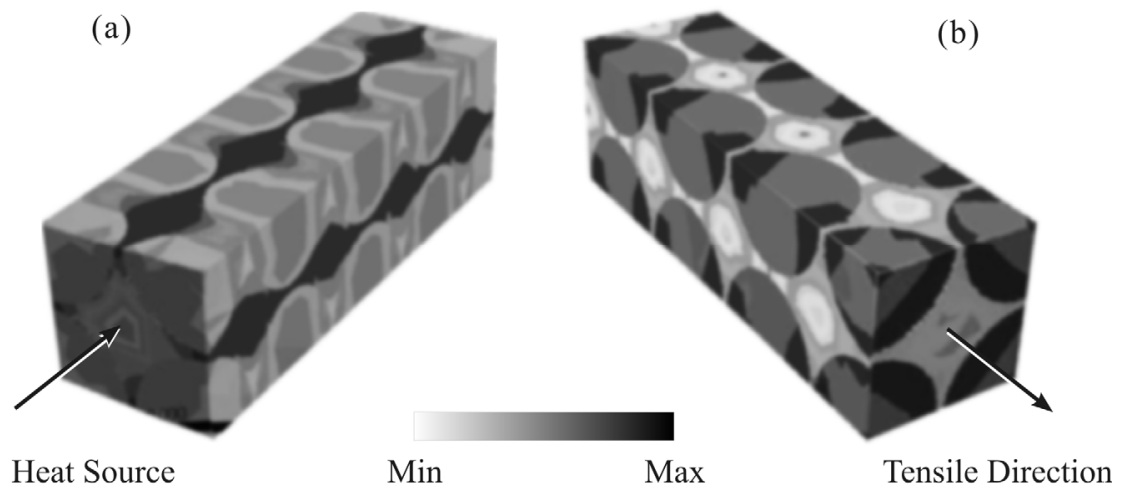

Figure 10: The computer graphics of simulated heat and stress distributions in the fabricated thermodynamic crystals with the body centered cubic structure. The graphics (a) and (b) show the heat and stress flows through the metal spheres and the ceramic matrix, respectively. 
mechanical properties of the artificial crystals. Figure 11 shows the simulated result of the temperature distribution in the graded body centered cubic structure composed of the copper spheres with the varied diameters form $1.75 \mathrm{~mm}$ to $1.9 \mathrm{~mm}$ in the alumina matrix. The heat flow transmitted from the source plane to the opposite side can be curved for the metal spheres arrangements with the larger diameter along the structural gradient. The thermodynamic crystals with the graded structures are considered to be applied to the effective heating and cooling devices in the novel mechanical tools, internal combustion engines and thermoelectric conversion elements along the artificial material designing.

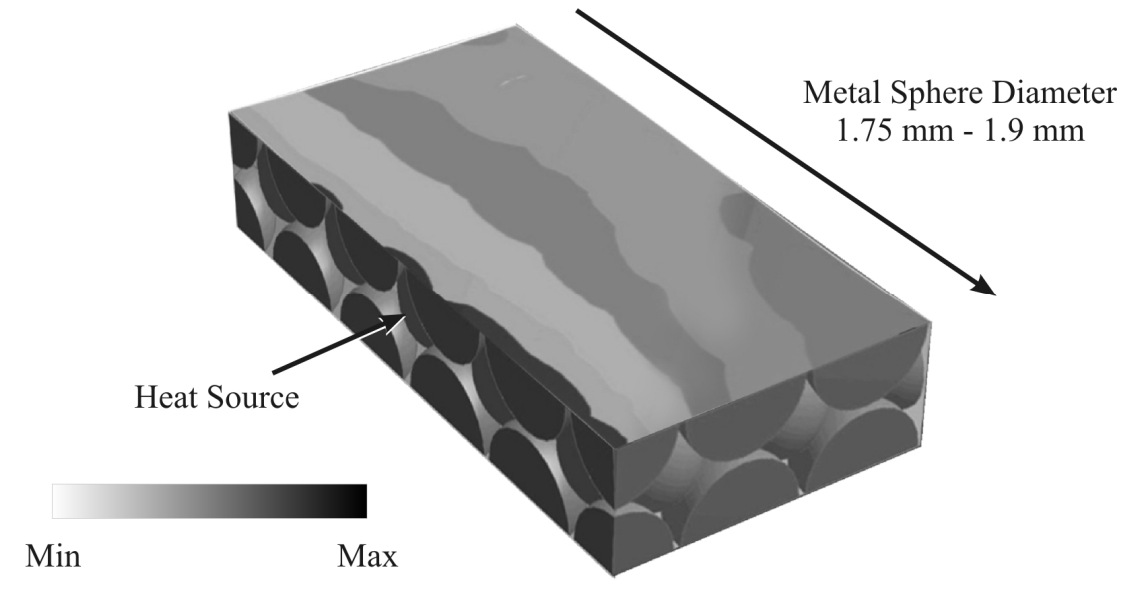

Figure 11: The simulated heat flow in the thermodynamic crystal of the graded structure with the body centered cubic pattern. The metal spheres with varied diameters were arranged in the ceramics matrix.

\section{Conclusions}

We have successfully fabricated photonic and thermodynamic crystals by using stereolithography and careful optimized dewaxing and sintering processes. The alumina photonic crystals with a diamond structure formed complete band gaps in terahertz wave frequencies. A twinned diamond crystals including with a plane defect between mirror symmetric lattice structures was also fabricated. A localized mode of a transmission peak was observed clearly in a forbidden band. In theoretical simulations of electromagnetic waves, localized mode profiles with multiple reflections were formed in a twinned interface. These micro dielectric components have strong potentials to be used as novel terahertz wave devices. Subsequently, the thermodynamic crystals with body centered cubic structures composed of pure copper spheres arrangements in alumina matrices were formed exactly. Heat and stress distributions in the crystals were simulated theoretically. The heat flows transmitted through the connected copper spheres with high thermal conductivities, and the tensile stresses were distributed periodically into the alumina lattices with high Young's modulus. Graded body centered cubic 
structures composed of the copper spheres with varied diameters in the alumina matrix were formed. The heat flow distributions could be curved along the graded direction for the copper spheres arrangements with the larger diameters. These artificial crystal structures are considered to be applied to the effective heating and cooling devices in the near future industrial fields.

\section{Acknowledgements}

This study was supported by Priority Assistance for the Formation of Worldwide Renowned Centers of Research - The Global COE Program (Project: Center of Excellence for Advanced Structural and Functional Materials Design) from the Ministry of Education, Culture, Sports, Science and Technology (MEXT), Japan.

\section{References}

[1] Ohtaka, K., Energy bang of photons and low energy photon diffraction. Physical Review B, 19(10), pp. 5057-5067, 1979.

[2] Yablonovitch \& E., Inhabited spontaneous emission in solid-state physics and electronics. Physical Review Letter, 58(20), pp. 2059-2062, 1987.

[3] Chen, W., Kirihara, S. \& Miyamoto, Y., Three-dimensional micro-photonic crystals of $\mathrm{ZrO}_{2}$ toughened $\mathrm{Al}_{2} \mathrm{O}_{3}$ for terahertz wave applications. Applied Physics Letter, 91(15), pp. 153507-1-3, 2007.

[4] Miyamoto, Y., Kanaoka. H. \& Kirihara, S., Terahertz wave localization at a three-dimensional ceramic fractal cavity in photonic crystals. Journal of Applied Physics, 103, pp. 103106-1-5, 2008.

[5] Van Exter, M., Fattinger, C. \& Grischkowsky, D., Terahertz time-domain spectroscopy of water vapour. Optics Letters, 14(20), pp. 1128-1130, 1989.

[6] Clery, D., Brainstorming their way to an imaging revolution. Science, 297, pp. 761- 763, 2002.

[7] Ho, K., Chan, C. \& Soukoulis, C., Existence of a photonic gap in periodic dielectric structures, Physical Review Letter, 65(25), pp. 3152-3165, 1990.

[8] Kirihara, S., Miyamoto, Y., Takenaga, K., Takeda, M. \& Kajiyama, K., Fabrication of electromagnetic crystals with a complete diamond structure by stereolithography. Solid State Communications, 121(8), pp. 435-439, 2002.

[9] Kirihara, S. \& Miyamoto, Y., Terahertz wave control by using ceramic photonic crystals with diamond structure including plane defects fabricated by micro-stereolithography. The International Journal of Applied Ceramic Technology, 6(1), pp. 41-44, 2009.

[10] Kirihara, S., Niki, T. \& Kaneko, M., Three-dimensional material tectonics for electromagnetic wave control by using micro-stereolithography. Ferroelectrics, 387, pp. 102-111, 2009.

[11] Kanaoka, H., Kirihara, S. \& Miyamoto, Y., Terahertz wave properties of alumina microphotonic crystals with a diamond structure. Journal of Materials Research, 23(4), pp. 1036-1041, 2008. 
114 Sustainable Chemistry

[12] Kirihara, S., Uehara, Y., \& Takinami Y., Fabrication of thermodynamic crystals by structural joining. Proc. of the 34th international Conference Advanced Ceramics and Composites, eds. T. Ohji \& M. Singh, John Wiley \& Sons: New Jersey, 31(8), pp. 169-174, 2010. 